\title{
SOLVABLE AUTOMORPHISM GROUPS AND AN UPPER BOUND FOR $|A(G)|$
}

\author{
J. R. WEAVER ${ }^{1}$
}

Aвstract. The objective of this work is to find a subgroup $H$ of a finite group $G$ which will give information about the order of the automorphism group of $G$ and the structure of the automorphism group of $G$. An upper bound is found for the order of the automorphism group and conditions are given which insure that the automorphism group is solvable. Some information is given about a normal subgroup of a particular subgroup of the automorphism group. In this paper all groups are assumed to be finite.

1.0. Notations. $H \leqq G, H \triangle G$, shall mean respectively, that $H$ is a subgroup, a normal subgroup of a group $G$.

$A(G)$ shall mean the automorphism group of $G$.

$\langle S\rangle=$ subgroup generated by the subset $S$ in $G$.

$N_{G}(H)=$ the normalizer of the subset $H$ in the group $G$.

$H^{x}=x^{-1} H x$ for $x$ in $G$.

$[G: H]=$ index of the subgroup $H$ in $G$.

$g^{\alpha}$ is the image of the element $g$ under the mapping $\alpha$.

$H^{\alpha}$ is the image of the subset under the mapping $\alpha$.

$F(G ; H)=\left\{\alpha \in A(G) \mid h^{\alpha}=h\right.$ for each $h$ in $\left.H\right\}$.

$|G|$ is the number of elements in the group $G$.

$I(G)$ shall mean the inner automorphism group of the group $G$.

1.1. Basic properties of $H$-automorphisms. Hans Liebeck introduced the notation for $H$-automorphism which is adopted.

Definition 1.1.1. Let $H \leqq G$. An automorphism $\alpha$ of $G$ is called an $H$-automorphism if $g^{-1} g^{\alpha} \in H$ for each $g$ in $G$. Denote the set of all $H$ automorphisms by $A(G ; H)$.

If $H \leqq G$, then $A(G ; H) \leqq A(G)$. Conversely, any subgroup $T$ of $A(G)$ can be regarded as a group of $H$-automorphisms for various choices of $H$. Since $A(G ; G)=A(G)$, one can always choose $H=G$.

Let $T \leqq A(G)$ for a group $G . K(G ; T)$ is used to represent $\left\langle g^{-1} g^{\alpha} \mid \alpha \in T, g \in G\right\rangle . K(G ; T)$ is called the multiplier subgroup of $T$. Liebeck [3] has shown that $T$ may be a proper subgroup of the group of all $K(G ; T)$-automorphisms. One easily shows that $K(G ; T) \triangle G$.

Received by the editors March 16, 1970.

AMS 1969 subject classifications. Primary 2022, 2025.

Key words and phrases. Finite group, automorphism group, solvable, upper bound.

1 These results are contained in the author's doctoral thesis which was written under the direction of Professor Joseph Adney at Michigan State University. 
The set of $\alpha \in A(G)$ such that $H^{\alpha}=H$ for $H$ a subgroup of $G$ is denoted by $\Gamma(G ; H) . \quad \Gamma(G ; H)$ is clearly a subgroup of $A(G)$ which contains $A(G ; H)$. This brings us to

Proposition 1.1.2. If $H \leqq G$, then $A(G ; H) \triangle \Gamma(G ; H)$.

Proof. Let $\alpha \in A(G ; H)$ and let $\gamma \in \Gamma(G ; H)$. If $g \in G$ then $g^{-1} g^{\gamma-1 \alpha \gamma}$ $=g^{-1}\left(g^{\gamma-1} h\right)^{\gamma}=h^{\gamma} \in H$ for some $h$ in $H$. Therefore $A(G ; H) \triangle \Gamma(G ; H)$.

Since $A(G ; H) \triangle \Gamma(G ; H)$, an obvious question to ask is, "What relationship exists between $\Gamma(G ; H), A(G ; H)$, and $K(G ; A(G ; H))$ ?" The following will give some information in this direction.

Lemma 1.1.3. If $H \leqq G$, then $K(G ; A(G ; H))$ is admissible with respect to $\Gamma(G ; H)$.

Proof. If $\gamma \in \Gamma(G ; H)$ and $g^{-1} g^{\alpha}$ is a generator of $K(G ; A(G ; H))$, then

$$
\left(g^{-1} g^{\alpha}\right)^{\gamma}=\left(g^{\gamma}\right)^{-1}\left(g^{\gamma}\right)^{\gamma-1 \alpha \gamma} \in K(G ; A(G ; H)) .
$$

Therefore $K(G ; A(G ; H))$ is admissible with respect to $\Gamma(G ; H)$.

THEOREM 1.1.4. If $H \leqq G$, then there exists a normal subgroup $M$ of $G$ such that $K(G ; A(G ; H)) \leqq M \leqq H, \Gamma(G ; H) / A(G ; H)$ is isomorphic to a subgroup of $A(G / M)$, and $M$ is admissible with respect to $\Gamma(G ; H) . M$ is also maximal with respect to being normal in $G$, being a subgroup of $H$, and being admissible with respect to $\Gamma(G ; H)$.

Proof. $K(G ; A(G ; H))$ is normal in $G$, is a subgroup of $H$, and is admissible with respect to $\Gamma(G ; H)$. Let $M$ be a subgroup of $G$ maximal with respect to these three properties.

Now define $\phi$ mapping $\Gamma(G ; H)$ into $A(G / M)$ by $\left(g^{\sigma}\right)^{(\gamma) \phi}=\left(g^{\gamma}\right)^{\sigma}$ where $\sigma$ is the natural homomorphism of $G$ onto $G / M$ and $\gamma \in \Gamma(G ; H)$. If $g^{\sigma}$ and $b^{\sigma}$ are two elements of $G / M$, then $\left(g^{\sigma} b^{\sigma}\right)^{(\gamma) \phi}=\left(g^{\sigma}\right)^{(\gamma) \phi}\left(b^{\sigma}\right)^{(\gamma) \phi}$.

It then follows that $(\gamma) \phi$ is an automorphism of $G / M$ for each $\gamma$ in $\Gamma(G ; H)$.

For $\gamma$ and $B$ in $\Gamma(G ; H),\left(g^{\sigma}\right)^{(\gamma B) \phi}=\left(g^{\sigma}\right)^{(\gamma) \phi(B) \phi}$. It follows from this that $\phi$ is a homomorphism of $\Gamma(G ; H)$ into $A(G / M)$.

The kernel of $\phi$ is $A(G ; H)$. Therefore $\Gamma(G ; H) / A(G ; H)$ is isomorphic to a subgroup of $A(G / M)$.

If $H \leqq G$, then $\Gamma(G ; H)^{\alpha}=\Gamma\left(G ; H^{\alpha}\right)$ and $A(G ; H)^{\alpha}=A\left(G ; H^{\alpha}\right)$ for $\alpha$ in $A(G)$.

$1.2 A$-intravariant and $A$-closed subgroups. Helmut W. Wielandt [4] calls a subgroup $H$ of $G$ intravariant in $G$ if and only if for each automorphism $\alpha$ in $A(G)$ there exists a $g$ in $G$ such that $\left(H^{\alpha}\right)^{g}=H$. This definition is generalized to give 
Definition 1.2.1. A subgroup $H$ of $G$ is $A$-intravariant in $G$ for $A$ a subgroup of $A(G)$ if and only if for each automorphism $\alpha \in A$ there exists a $g$ in $G$ such that $\left(H^{\alpha}\right)^{o}=H$.

Some of the better known $A(G)$-intravariant subgroups are Sylow subgroups and characteristic subgroups of any group $G$; and Hall subgroups, Carter subgroups, and system normalizers of solvable groups.

Proposition 1.2.2. If $H \leqq G$, then $H$ is $A$-intravariant in $G$ for $A$ a subgroup of $A(G)$ if and only if $A \leqq \Gamma(G ; H) I(G)$.

Proof. Since $I(G) \triangle A(G), \Gamma(G ; H) I(G)$ is a well-defined subgroup of $A(G)$. If $H$ is $A$-intravariant then for each $\alpha \in A$ there exist a $g$ in $G$ such that $\left(H^{\alpha}\right)^{\circ}=H$. Therefore $\alpha \in \Gamma(G ; H) I(G)$ and $A \leqq \Gamma(G ; H) I(G)$

If $A \leqq \Gamma(G ; H) I(G)$ then each element in $A$ may be represented as the product of an element from $\Gamma(G ; H)$ and an element from $I(G)$. Therefore $H$ is $A$-intravariant.

Upon considering the subgroup $\Gamma(G ; H) \cap I(G)$ for $H \leqq G$, it is easily shown that $\Gamma(G ; H) \cap I(G) \cong N_{G}(H) / Z(G)$. This leads to

Proposition 1.2.3. Let $H \leqq G$ and assume that $I(G) \leqq A \leqq A(G)$. Then $H$ is $A$-intravariant if and only if $\left[G: N_{G}(H)\right]=[A: A \cap \Gamma(G ; H)]$.

Proof. Since $A \leqq \Gamma(G ; H) I(G)$,

$$
A=A \cap \Gamma(G ; H) I(G)=I(G)(A \cap \Gamma(G ; H)) .
$$

Hence

$$
|A|=|I(G)||A \cap \Gamma(G ; H)| /|\Gamma(G ; H) \cap I(G)| .
$$

Therefore $[A: A \cap \Gamma(G ; H)]=\left[G: N_{G}(H)\right]$.

Conversely; suppose that $\left[G: N_{G}(H)\right]=[A: A \cap \Gamma(G ; H)]$. Since $I(G) \leqq A, I(G)(A \cap \Gamma(G ; H)) \leqq A$. Note that $|A|=|I(G)(A \cap \Gamma(G ; H))|$. Hence $A=I(G)(A \cap \Gamma(G ; H))=A \cap \Gamma(G ; H) I(G)$. Therefore $A \leqq$ $\Gamma(G ; H) I(G)$ and $H$ is $A$-intravariant.

It is easy to show that if $H$ is $A$-intravariant in $G$, then $N_{G}(H)$ is $A$ intravariant in $G$ for $A$ a subgroup of $A(G)$.

In 1937 Philip Hall [2] showed that if $G$ is a solvable group, then the Sylow system $S$ is $A(G)$-intravariant. Then he showed that $A(G)$ $=I(G) \Gamma(G ; S)$. With this he was able to arrive at an upper bound for the order of $A(G)$. Later on an upper bound for $|(A G)|$ will be found.

1.3. $A$-closed subgroups. Recall that $H$ is weakly closed in the subgroup $K$ if $H^{0} \leqq K \leqq G$ implies that $H^{o}=H$ for $g$ in $G$.

Definition 1.3.1. A subgroup $H$ of a group $G$ is $A$-closed in $G$ for $A$ a subgroup of $A(G)$ if: 
(i) $H$ is $A$-intravariant in $G$, and,

(ii) $H$ is weakly closed in $N_{G}(H)$.

Proposition 1.3.2. If $H \leqq G$, then $A(G ; H) \leqq \bigcap_{o \in G} \Gamma\left(G ; H^{*}\right)$ $\leqq A\left(G ; N_{G}(H)\right)$.

Proof. Assume that $\alpha \in A(G ; H)$. It then follows that $\left(h^{a}\right)^{-1}\left(h^{a}\right)^{\alpha}$ $\in K(G ; A(G ; H)) \triangle G$. Therefore $K(G ; A(G ; H)) \leqq H^{o}$ and $\left(h^{o}\right)^{\alpha} \in H^{\boldsymbol{\theta}}$.

Suppose that $\alpha \in \bigcap_{o \in G} \Gamma\left(G ; H^{o}\right)$. If $g \in G$ and $h \in H$, then $h^{\sigma^{-1}}$ $=\left(x^{\theta^{-1}}\right)^{\alpha}$ for some $x$ in $H$. On the other hand, $\left(x^{\sigma^{-1}}\right)^{\alpha}=\left(x^{\alpha}\right)^{\left(\sigma^{\alpha}\right)^{-1}}$ $=h^{g^{-1}}$. Hence $x^{\alpha}=h^{\sigma^{-1} g^{\alpha}}$ is an element of $H$ and $g^{-1} g^{\alpha} \in N_{G}(H)$. Therefore $\alpha \in A\left(G ; N_{G}(H)\right)$. It is useful to know when $\bigcap_{o \in G} \Gamma\left(G ; H^{q}\right)$ $=A\left(G ; N_{G}(H)\right)$. This brings us to

Proposition 1.3.3. If $H$ is a $\Gamma\left(G ; N_{G}(H)\right)$-closed subgroup of $G$, then $A\left(G ; N_{G}(H)\right)=\bigcap_{o \in G} \Gamma\left(G ; H^{o}\right)$.

Proof. By 1.3.2, $\bigcap_{o \in G} \Gamma\left(G ; H^{o}\right) \leqq A\left(G ; N_{G}(H)\right) \leqq \bigcap_{o \in G} \Gamma\left(G ; N_{G}(H)_{o}\right)$ It is easily shown that $\Gamma\left(G ; H^{o}\right)=\Gamma\left(G ; N_{G}(H)^{g}\right)$ for each $g$ in $G$. Therefore $A\left(G ; N_{G}(H)\right)=\bigcap_{o \in G} \Gamma\left(G ; N_{G}(H)^{\circ}\right)$.

The converse of 1.3.3. is not true. Let $H$ be the subgroup of the alternating group of degree 5 which is equal to $\langle(1,2)(3,4)\rangle . N_{G}(H)$ $=\langle(1,2)(3,4),(1,3)(2,4)\rangle$ and it can be shown that $H$ is not weakly closed in $N_{G}(H)$.

1.4. $A(G)$-closed and $A(G)$-intravariant subgroups. In this section the existence of an $A(G)$-closed subgroup $H$ in the group $G$ will be used to a establish the structure of $A(G)$.

Lemma 1.4.1. If $H$ is an $A(G)$-intravariant subgroup of $G$, then $A(G)$ is solvable if and only if $\Gamma(G ; H) / \Gamma(G ; H) \cap I(G)$ and $G$ are solvable.

Proof. If $A(G)$ is solvable then it is obvious that $\Gamma(G ; H) / \Gamma(G ; H)$ $\cap I(G)$ and $G$ are solvable.

Conversely, suppose that $G$ and $\Gamma(G ; H) / \Gamma(G ; H) \cap I(G)$ are solvable. By 1.2.2 $A(G)=\Gamma(G ; H) I(G)$. Hence

$$
A(G) / I(G) \cong \Gamma(G ; H) / \Gamma(G ; H) \cap I(G) .
$$

Since $G$ is solvable, $I(G)$ is solvable and $A(G)$ is solvable.

THEOREM 1.4.2. Suppose that $H$ is $A(G)$-closed in the solvable group $G$ with $\Gamma(G ; H)$ a maximal subgroup of $A(G)$. If $\Gamma(G ; H) / \Gamma(G ; H)$ $\cap I(G)$ is solvable with $A\left(G ; N_{G}(H)\right) \cong\langle 1\rangle$, then $A(G)$ is solvable and there exists a minimal normal subgroup $T$ of $A(G)$ and $A(G)$ satisfies the following:

(i) $C_{A(G)}(T)=T$; 
(ii) $T$ is the minimal normal subgroup of $A(G)$;

(iii) $A(G)=\Gamma(G ; H) T$;

(iv) $T \cap \Gamma(G ; H) \cong\langle 1\rangle$;

(v) $|T|=[A(G): \Gamma(G ; H)]$;

(vi) if $G_{0} / T$ is a minimal normal subgroup of $A(G) / T$ and $A_{0}$ $=\Gamma(G ; H) \cap G_{0}$, then $\Gamma(G ; H)=N_{A(G)}\left(A_{0}\right),\left|G_{0}\right|=|T|\left|A_{0}\right|,|T|=p^{a}$, $\left|A_{0}\right|=q^{b}$ and $p \neq q$ for primes $p$ and $q$ and nonnegative integers $a$ and $b$.

Proof. By 1.4.1, $A(G)$ is a solvable group. Hence $A(G)$ has a minimal normal subgroup $T$.

Note that $A\left(G ; N_{G}(H)\right)=\bigcap_{o \in G} \Gamma\left(G ; H^{\theta}\right)=\bigcap_{\alpha \in A(G)} \Gamma(G ; H)^{\alpha}$. It follows from [4] that $A(G)=\Gamma(G ; H) T$ where $T$ is the minimal normal subgroup of $G$. Then $C_{A(G)}(T)=T, \Gamma(G ; H) \cap T \cong\langle 1\rangle,[A(G): \Gamma(G ; H)]$ $=T$, and condition (vi) is satisfied.

The condition that $\Gamma(G ; H)$ be a maximal subgroup of $A(G)$ could be replaced by $\left[G: N_{G}(H)\right]$ is a prime number. Then $|T|=\left[G: N_{G}(H)\right]$.

Continuing in the search for groups whose automorphism groups are solvable we arrive at

Proposition 1.4.3. Let each subgroup in a composition series of the subgroup $H$ of the solvable group $G$ be $\Gamma(G ; H)$-closed for $H$ an $A(G)$ intravariant subgroup of $G$. If $F(G ; H)$ is solvable then $A(G)$ is solvable.

Proof. By 1.4.1, $A(G)$ is solvable if $\Gamma(G ; H) / \Gamma(G ; H) \cap I(G)$ is solvable. Note that $F(G ; H) \triangle \Gamma(G ; H)$.

Let $\langle 1\rangle=N_{r} \leqq N_{r-1} \leqq \cdots \leqq N_{0}=H$ be the composition series of $H$ mentioned in the hypothesis. Using induction it is easy to show that each $N_{i} \triangle H$ for $i=0,1, \cdots, r$. If $\alpha \in \Gamma(G ; H)$, then $N_{i}^{\alpha}=N_{i}^{o} \leqq H$ for some $g$ in $G$. Since $N_{i}^{o} \leqq H \leqq N_{G}(H)$ we have that $N_{i}^{\alpha}=N_{i}$. Therefore $\Gamma(G ; H)$ leaves each subgroup in the series fixed. Note that $\Gamma(G ; H) / F(G ; H)$ is isomorphic to a subgroup of $A(H)$. By Gaschütz $[1], \Gamma(G ; H) / F(G ; H)$ is solvable, $\Gamma(G ; H)$ is solvable and the proof is complete.

1.5. An upper bound for the order of $A(G)$. An upper bound for $|A(G)|$ will be derived by using the concept of $H$ being $A(G)$ intravariant in $G$.

THEOREM 1.5.1. Let $H$ be an $A(G)$-intravariant subgroup of $G$ with $F\left(G ; K_{1}\right) \leqq A(G ; H)$ where $K_{1}=K(G ; A(G ; H))$. Then $|A(G)|$ divides $\left[G: N_{G}(H)\right]\left|A\left(K_{1}\right)\right|\left|F\left(G ; K_{1}\right)\right|$. The prime divisors of $\left|F\left(G ; K_{1}\right)\right|$ are prime divisors of $\left|K_{1}\right|$.

Proof. If $p$ is a prime dividing $\left|F\left(G ; K_{1}\right)\right|$ then there exists an $\alpha$ in $F\left(G ; K_{1}\right)$ such that $|\alpha|=p$. Since $\alpha$ is not the identity, there exists a $g$ 
in $G$ such that $g^{-1} g^{\alpha}=k \in K_{1}, k \neq 1$. Hence $g^{\alpha^{p}}=g k^{p}=g$. Therefore $k^{p}=1$ and $p$ divides $\left|K_{1}\right|$.

By 1.1.3, $K_{1}$ is admissible with respect to $\Gamma(G ; H)$. Define $\phi$ mapping $\Gamma(G ; H)$ into $A\left(K_{1}\right)$ by $(k)^{(\gamma) \phi}=k^{\gamma}$ for each $k$ in $K_{1} . \phi$ is a homomorphism of $\Gamma(G ; H)$ into $A\left(K_{1}\right)$ and the kernel of $\phi$ is $F\left(G ; K_{1}\right)$. Therefore $\Gamma(G ; H) / F\left(G ; K_{1}\right)$ is isomorphic to a subgroup of $A\left(K_{1}\right)$.

It follows from 1.2.3 that $[A(G): \Gamma(G ; H)]=\left[G: N_{G}(H)\right]$. Since $\left|\Gamma(G ; H) / F\left(G ; K_{1}\right)\right|$ divides $\left|A\left(K_{1}\right)\right|,|A(G)|$ divides $\left[G: N_{G}(H)\right]$ $\left|A\left(K_{1}\right)\right|\left|F\left(G ; K_{1}\right)\right|$.

We are now in a position to prove

CoROllary 1.5.2. Let $H$ be a characteristic subgroup of $G$ such that $F\left(G ; K_{1}\right) \leqq A(G ; H)$ where $K_{1}=K(G ; A(G ; H))$ is a cyclic p-group of $G$, then $|A(G)|$ divides $p^{m}(p-1)$ for some nonnegative integer $m$.

Proof. If $\left|K_{1}\right|=2^{t}$ then $|A(G)|$ divides some power of 2 .

Assume that the order of $K_{1}$ is equal to $p^{n}$ for an odd prime $p$. Then $\left|A\left(K_{1}\right)\right|=p^{n-1}(p-1)$ and $|A(G)|$ divides $p^{m}(p-1)$ for some nonnegative in teger $m$.

COROLlaRy 1.5.3. Let $H$ be a characteristic subgroup of $G$ such that $K_{1}=K(G ; A(G ; H))$ is a cyclic p-group with $p$ a prime of the form $2^{m}+1$ for some integer $m$. If $F\left(G ; K_{1}\right) \leqq A(G ; H)$ then $|A(G)|$ divides $p^{n} 2^{m}, A(G)$ is solvable, and $G$ is solvable.

Proof. By 1.5.2, $|A(G)|$ divides $p^{n} 2^{m}$ for some integer $n$. Hence $A(G)$ and $G$ are solvable.

COROllary 1.5.4. If $H$ is a normal Sylow 2-subgroup of $G$ such that $F\left(G ; K_{1}\right) \leqq A(G ; H)$ for $K_{1}=K(G ; A(G ; H))$ cyclic, then $G=H$.

Proof. By 1.5.2, $|A(G)|=2^{n}$ for some integer $n$. Hence $A(G)$ is a nilpotent group and $G$ is nilpotent.

Assume that there exists a prime $p \neq 2$ which divides $|G|$. Since $G$ is a nilpotent group, $A(G)$, is a direct product of the automorphism groups of the Sylow subgroups of $G$.

Let $\alpha \in A\left(H_{p}\right)$ where $H_{p}$ is the Sylow $p$-subgroup of $G$. Note that $\alpha$ can be thought of as an element in $A(G)$ that acts as the identity on $H$ and is an element of $A(G ; H)$. If $g \in H_{p}$ then $g^{-1} g^{\alpha} \in H \cap H_{p}=\langle 1\rangle$. Therefore $\alpha$ is the identity on $H_{p}$ and $A\left(H_{p}\right) \cong\langle 1\rangle$. Since $2<p$, we have a contradiction. Therefore $H=G$.

The upper bound for $|A(G)|$ found in 1.5.1 is actually attained if $G$ is the symmetric group of degree 3 and $H$ is the alternating group of degree 3. 


\section{REFERENCES}

1. W. Gaschütz, Gruppen, in denen das Normalteilersein transitiv ist, J. Reine Angew. Math. 198 (1957), 87-92. MR 19, 940.

2. P. Hall, On the Sylow systems of a solvable group, Proc. London Math. Soc. 43 (1937), 316-323.

3. H. Liebeck, The automorphism group of finite p-groups, J. Algebra 4 (1966), 426-432. MR 34 \#653.

4. W. Wielandt, Topics in the theory of composite groups, Lecture Notes, Department of Mathematics, University of Wisconsin, Madison, Wis., 1967.

Michigan State University, East Lansing, Michigan 48823

The University of West Florida, Pensacola, Florida 32504 